wave bandpass filters by standard $0.18-\mu \mathrm{m}$ CMOS technology, IEEE Electron Device Lett 28 (2007), 220-222.

6. J. Brinkhoff and F. Lin, Integrated filters for $60 \mathrm{GHz}$ systems on CMOS, International Workshop on Radio-Frequency Integration Technology, 2007.

7. C.-Y. Hsu, C.-Y. Chen, and H.-R. Chuang, A $60-\mathrm{GHz}$ millimeter-wave bandpass filter using $0.18-\mu \mathrm{m}$ CMOS technology, IEEE Electron Device Lett 29 (2008), 246-248.

8. S.B. Cohn, Dissipation loss in multiple-coupled-resonator filters, Proc IRE 47 (1959), 1342-1348.

9. G.L. Matthaei, L. Young, and E.M.T. Jones, Microwave filters, impedance-matching networks, and coupling structures, Artech House, Dedham, MA, 1980.

10. Sonnet EM Suite, Sonnet Software Inc., NY, 2003.

(C) 2008 Wiley Periodicals, Inc.

\section{CHARACTERISTICS OF GaAs TRANSFORMERS FOR RFIC APPLICATIONS}

\section{Chinchun Meng, Ya-Hui Teng, Jin-Siang Syu, Yi-Chen Lin,} Jhin-Ci Jhong, and Ying-Chieh Yen

Department of Communication Engineering, National Chiao Tung University, Hsinchu, Taiwan, Republic of China; Corresponding

author: ccmeng@mail.nctu.edu.tw

\section{Received 18 March 2008}

ABSTRACT: In this article, stacked transformers, coupling stacked transformers, interleave transformers, and symmetrical transformers on GaAs substrate are systematically studied. Two kinds of stacked transformer are under study. One has the symmetrical electric property while the other one has a better quality factor for the primary port. The stack transformers have achieved the highest coupling coefficient $(\sim 0.9)$ at the cost of lower self-resonance frequency. The interleave transformers have the identical electrical properties for the primary and the secondary ports. However, the layout is incompatible with the differential operation. On the other hand, the symmetrical transformer is compatible with the differential operation and can have the center-tapped biasing option. The data established here provides a useful design library for the GaAs RFIC. () 2008 Wiley Periodicals, Inc. Microwave Opt Technol Lett 50: 2937-2942, 2008; Published online in Wiley InterScience (www. interscience.wiley.com). DOI 10.1002/mop.23853

Key words: GaAs; transformer; stack transformer; interleave transformer; symmetrical transformer

\section{INTRODUCTION}

Transformers have been widely employed in the silicon RFIC design [1-7]. However, not too much study has been done along the direction of GaAs transformers even though GaAs technologies entered the $\mathrm{GHz}$ regime well before the silicon technology. One possible reason is that because GaAs has the semi-insulating substrate, and the impedance matching design method can be applied straightforwardly to maximize the circuit performance such as the power gain for amplifiers. Thus, various transmission lines on semi-insulating GaAs substrate have been widely used and characterized in the past and the single-ended circuit topology prevails in the guided-wave impedance matching design methodology. The advanced silicon IC technology driven by the aggressively silicon device scaling has made possible the silicon RFICs. Because silicon has lossy substrate, the differential operation is inevitable to keep the signal integrity at the $\mathrm{GHz}$ regime. Thus, many differentially operated analog circuits such as Gilbert mixers

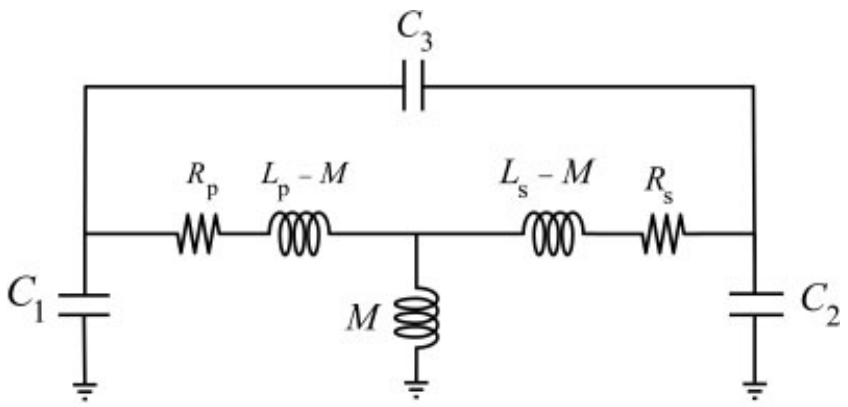

Figure 1 The equivalent circuit model of GaAs transformer

and cross-coupled oscillators have been introduced in the silicon RFIC design [8-10]. The transformer which serves as the important ingredient for the differential operation has gained its role in the RFIC design.

Recently, the analog circuit design approach at the $\mathrm{GHz}$ regime has also gained its ground in the GaAs-based technology [11]. The GaInP/GaAs HBT Gilbert mixers exhibit excellent performance. Low-IF Hartley receiver with polyphase filters, dual conversion Weaver receiver, and subharmonic direct conversion receiver have also been implemented by the GaInP/GaAs HBT technology [1215]. Transformer-based GaInP/GaAs Gilbert mixers have been demonstrated for the low-voltage operation at high frequencies [5] [16]. The state-of-the-art transformer oscillators have also been demonstrated in GaInP/GaAs HBT technology [17]. Apparently, there is a need to perform a detailed study for RF transformer on GaAs substrate.

\section{GaAs TRANSFORMER MODEL}

A transformer equivalent circuit model suitable for GaAs is shown in Figure 1. $L_{\mathrm{p}}$ is the primary port self-inductance, $L_{\mathrm{s}}$ is the secondary port self-inductance, and $M$ is the mutual inductance. $R_{\mathrm{p}}$ and $R_{\mathrm{s}}$ are the series metal resistances of the primary winding and the secondary winding, respectively. $\mathrm{C} 1$ is the parasitic capacitance between the primary winding and the ground, $\mathrm{C} 2$ is parasitic capacitance between the secondary winding and the ground, and $\mathrm{C} 3$ is the capacitance between two windings. The GaAs transformer equivalent circuit is distinguished from the silicon transformer model in two aspects. First of all, the absence of resistors in parallel with $\mathrm{C} 1$ and $\mathrm{C} 2$ is evident by the fact that GaAs has a semi-insulating substrate. Second, the $\mathrm{C} 1$ and $\mathrm{C} 2$ are larger because of the standard GaAs process has a final substrate thickness of $100 \mu \mathrm{m}$ to facilitate the GaAs microstrip line. At low frequencies, all the parasitic capacitance can be neglected. Thus, the following equations can be employed to evaluate the inductances and resistance.

$$
\begin{gathered}
L_{p}=\frac{\operatorname{Im}\left[Z_{11}\right]}{2 \pi f} \quad R_{p}=\operatorname{Re}\left[Z_{11}\right] \\
L_{s}=\frac{\operatorname{Im}\left[Z_{22}\right]}{2 \pi f} \quad R_{s}=\operatorname{Re}\left[Z_{22}\right] \quad M=\frac{\operatorname{Im}\left[Z_{21}\right]}{2 \pi f}
\end{gathered}
$$

The quality factors for the primary winding and the secondary winding and the coupling factor can thus be defined by the following equations.

$$
Q_{p}=\frac{\operatorname{Im}\left[Z_{11}\right]}{\operatorname{Re}\left[Z_{11}\right]} \quad Q_{s}=\frac{\operatorname{Im}\left[Z_{22}\right]}{\operatorname{Re}\left[Z_{22}\right]} \quad K=\frac{M}{\sqrt{L_{p} L_{s}}}
$$




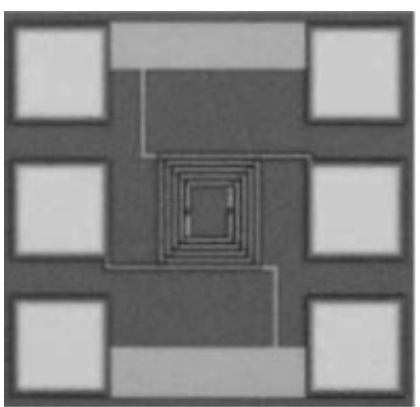

(a)

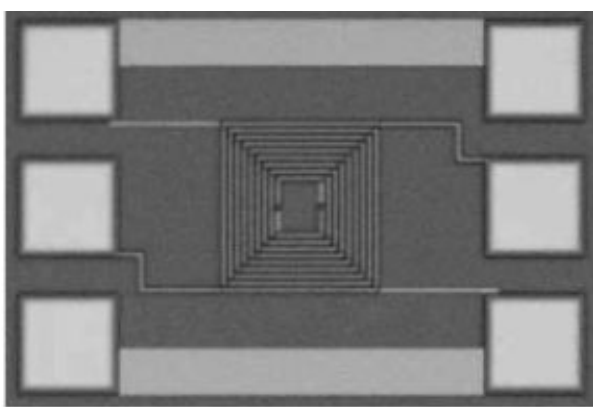

(b)

Figure 2 Photos of 5:5 and 9:9 stacked transformers

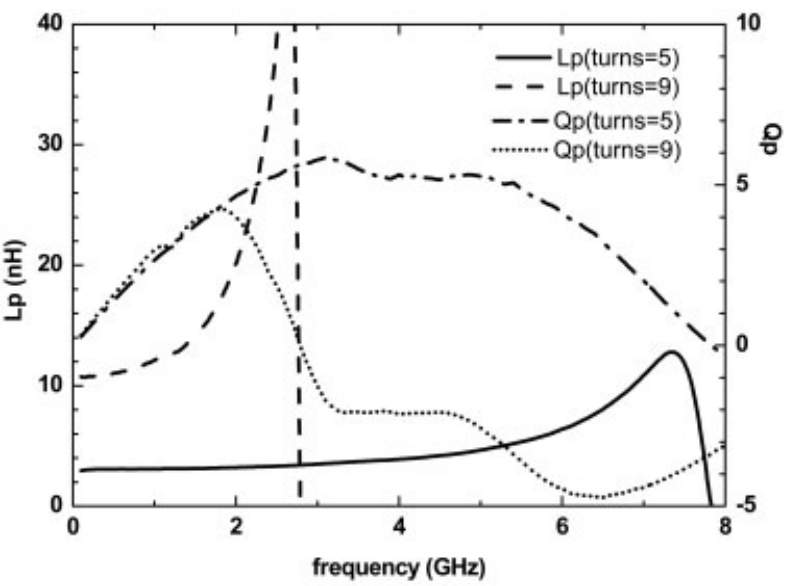

(a)

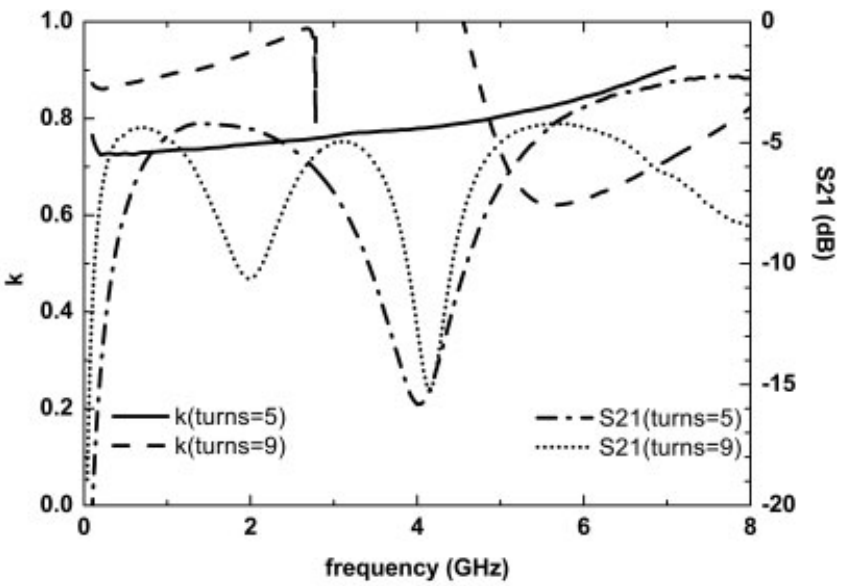

(b)

Figure 3 (a) Quality factor and inductance (b) coupling coefficient and transmission gain as a function of frequencies for 5:5 and 9:9 stacked transformer

TABLE 1 Stacked Transformer Results

\begin{tabular}{lccccc}
\hline Type & $L_{\mathrm{p}}$ at $1 \mathrm{GHz}(\mathrm{nH})$ & $R_{\mathrm{p}}$ at $1 \mathrm{GHz}(\mathrm{Ohm})$ & $Q_{\mathrm{pmax}}$ & $F$ at $Q_{\mathrm{pmax}}(\mathrm{GHz})$ & $\mathrm{SRF}(\mathrm{GHz})$ \\
\hline Turns $=5: 5$ & 3.09 & 7.293 & 5.853 & 3.1 & 7.83 \\
Turns $=9: 9$ & 12.11 & 25.615 & 4.255 & 1.8 & 2.79 \\
\hline
\end{tabular}

Thus, quality factor, inductance, and coupling coefficient can be plotted as a function of frequency once the transformer's $S$ parameters are measured without exploring the equivalent circuit model.

\section{VARIOUS GaAs TRANSFORMERS}

The GaAs process employed here has only two interconnect metal layers. The first metal is $1 \mu \mathrm{m}$ in thickness, the second metal is 1.6 $\mu \mathrm{m}$ in thickness, and the intermetal dielectric polyimide is $1.6 \mu \mathrm{m}$ in thickness.

\subsection{Stacked Transformers}

Photos of two stacked transformers (5:5 and 9:9) are shown in Figure 2. The primary winding consists of both Metal 1 and Metal 2. The metal width is $5 \mu \mathrm{m}$ and the spacing is $2.5 \mu \mathrm{m}$, respectively. It is obvious that the primary winding is identical to the secondary winding. Thus, only the quality factor and inductance for the primary winding are shown in Figure 3(a). The coupling coefficient and transmission gain are also shown in Figure 3(b). The coupling coefficients at $1 \mathrm{GHz}$ for the 5:5 and 9:9 stacked transformers are 0.74 and 0.89 , respectively. Table 1 summarizes the results.

\subsection{Coupling Stacked Transformers}

The vertically coupled stacked transformers employed by Zannoth et al. [6], which require only two metal layers, are used here. The fabricated 3:3 and 4:4 coupling stacked transformers with square spiral shape are shown in Figure 4. The primary side of the transformer and the secondary side of the transformer is formed by the top metal and by the bottom metal, respectively. The loss for the primary winding is minimized because the top metal is thicker. The turn-to-turn connections are made in the gap between the adjacent metal lines. The metal width is $8 \mu \mathrm{m}$ and the spacing is $17 \mu \mathrm{m}$, respectively. The quality factor and inductance for the primary winding and the secondary winding are shown in Figures 5(a) and 5(b). The coupling coefficient and transmission gain are also shown in Figure 5(c). The coupling coefficients at $1 \mathrm{GHz}$ for the $3: 3$ and $4: 4$ stacked transformers are 0.55 and 0.83 , respectively. Table 2 summarizes the results.

\subsection{Interleave Transformers}

Photos of two interleave transformers (4:4 and 5:5) are shown in Figure 6. Metal 2 is used for the winding while Metal 1 is used as the underpass. The metal width is $3.2 \mu \mathrm{m}$ and the spacing is 3.8 


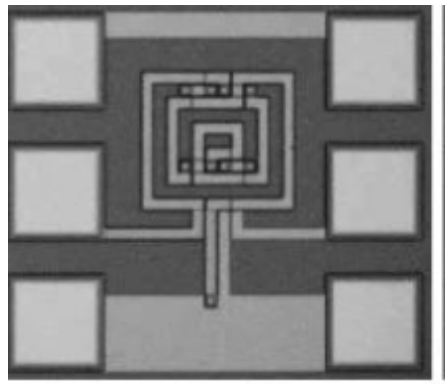

(a)

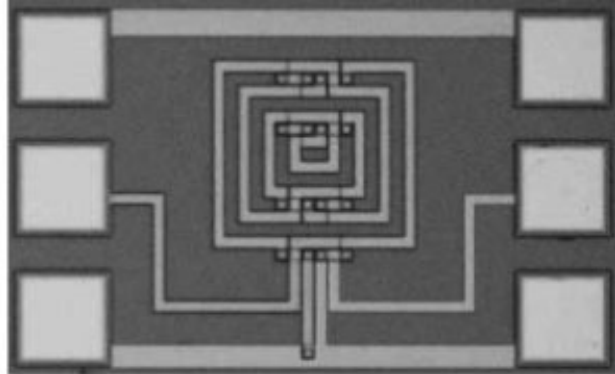

(b)

Figure 4 Photos of 3:3 and 4:4 coupling stacked transformers

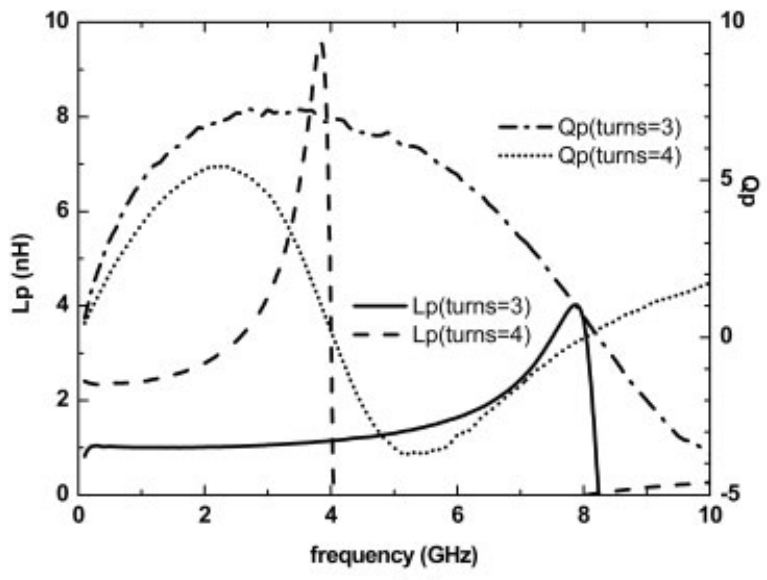

(a)

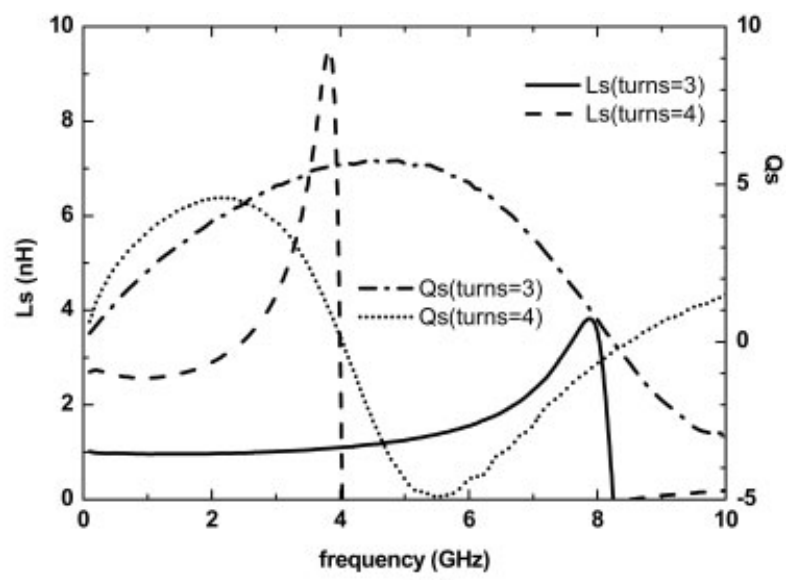

(b)

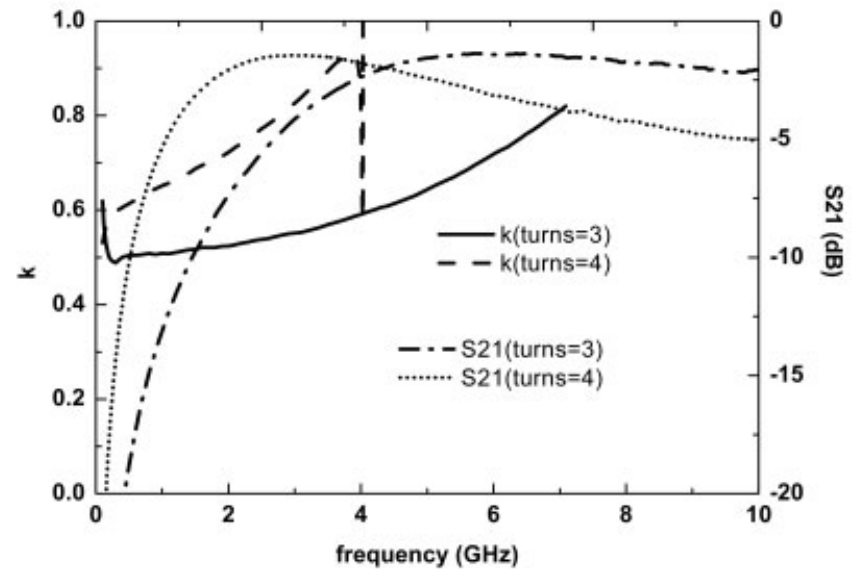

(c)

Figure 5 (a) Quality factor and inductance for the primary winding, (b) quality factor and inductance for the secondary winding, (c) coupling coefficient and transmission gain as a function of frequencies for $3: 3$ and $4: 4$ coupling stacked transformers

TABLE 2 Coupling Stacked Transformer Results

\begin{tabular}{|c|c|c|c|c|c|}
\hline Type & $L_{\mathrm{p}}$ at $1 \mathrm{GHz}(\mathrm{nH})$ & $R_{\mathrm{p}}$ at $1 \mathrm{GHz}(\mathrm{Ohm})$ & $Q_{\text {pmax }}$ & $F$ at $Q_{\text {pmax }}(\mathrm{GHz})$ & $\mathrm{SRF}(\mathrm{GHz})$ \\
\hline $3: 3$ & 1.007 & 1.285 & 7.299 & 3.5 & 8.24 \\
\hline \multirow[t]{2}{*}{$4: 4$} & 2.399 & 4.220 & 5.439 & 2.3 & 4.04 \\
\hline & $L_{\mathrm{s}}$ at $1 \mathrm{GHz}(\mathrm{nH})$ & $R_{\mathrm{s}}$ at $1 \mathrm{GHz}(\mathrm{Ohm})$ & $Q_{\text {smax }}$ & $F$ at $Q_{\text {smax }}(\mathrm{GHz})$ & $\mathrm{SRF}(\mathrm{GHz})$ \\
\hline $3: 3$ & 0.962 & 2.701 & 5.752 & 4.9 & 8.25 \\
\hline $4: 4$ & 2.566 & 4.617 & 4.575 & 2.1 & 4.03 \\
\hline
\end{tabular}




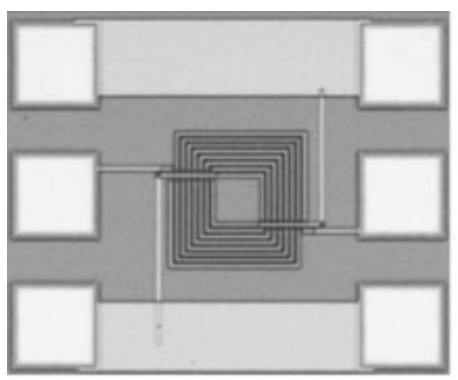

(a)

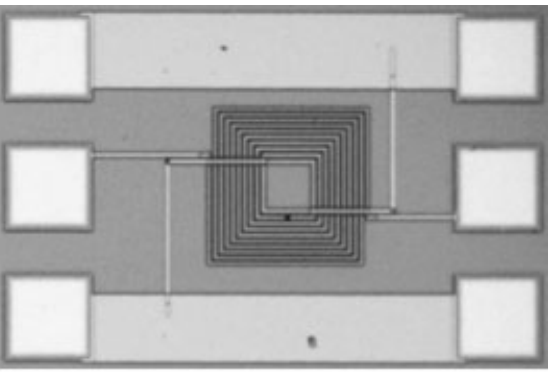

(b)

Figure 6 Photos of 4:4 and 5:5 interleave transformers

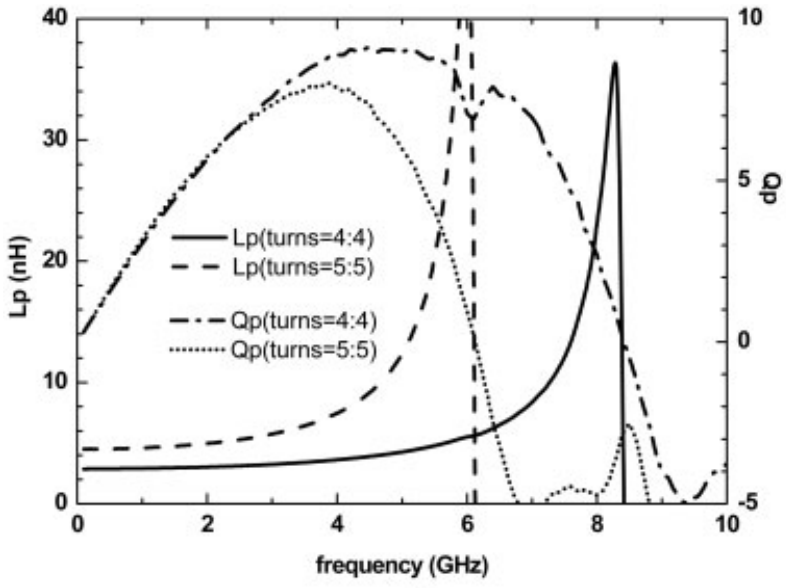

(a)

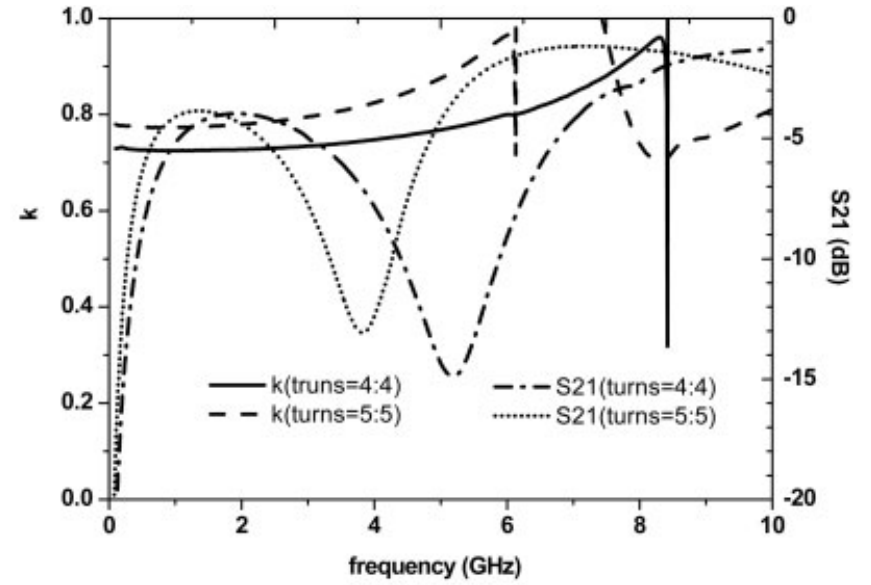

(b)

Figure 7 (a) Quality factor and inductance (b) coupling coefficient and transmission gain as a function of frequencies for 4:4 and 5:5 interleave transformer TABLE 3 Interleave Transformer Results

\begin{tabular}{lccccc}
\hline Type & $L_{\mathrm{p}}$ at $3 \mathrm{GHz}(\mathrm{nH})$ & $R_{\mathrm{p}}$ at $3 \mathrm{GHz}(\mathrm{Ohm})$ & $Q_{\mathrm{pmax}}$ & $F$ at $Q_{\mathrm{pmax}}(\mathrm{GHz})$ & \multicolumn{2}{c}{$\mathrm{SRF}(\mathrm{GHz})$} \\
\hline $4: 4$ & 3.242 & 8.065 & 9.143 & 4.5 & 8.42 \\
$5: 5$ & 5.75 & 14.775 & 8.022 & 3.9 & 6.13 \\
\hline
\end{tabular}

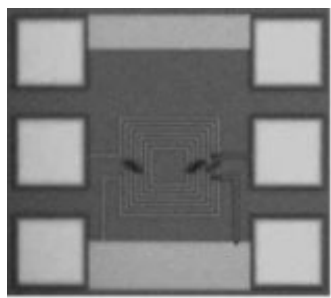

I

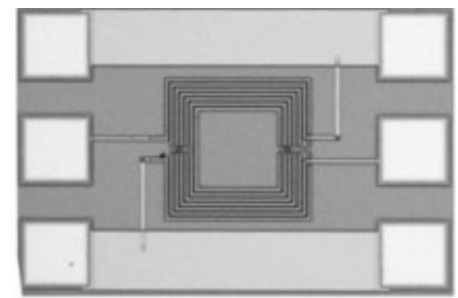

II

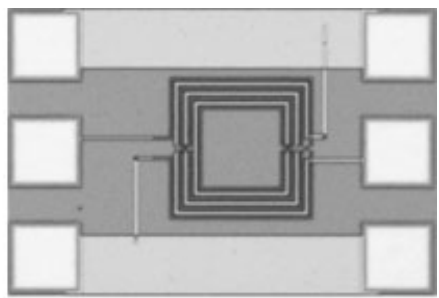

III

Figure 8 Photos of 4:3 symmetrical transformers

$\mu \mathrm{m}$, respectively. It is obvious that the primary winding is identical to the secondary winding. Thus, only the quality factor and the inductance for the primary winding are shown in Figure 7(a).

The coupling coefficient and transmission gain are also shown in Figure 7(b). The coupling coefficients at $1 \mathrm{GHz}$ for the $4: 4$ and 5:5 interleave transformers are 0.77 and 0.88 , respectively. Table 3 summarizes the results.

\subsection{Symmetrical Transformers}

Photos of three 4:3 symmetrical transformers are shown in Figure 8. Metal 2 is used for the winding while Metal 1 is used as the underpass. The metal width is $3.8 \mu \mathrm{m}$ and the spacing is $3.8 \mu \mathrm{m}$ for the first two symmetrical transformers. The inner diameter is smaller for the first one. The second symmetrical transformer has the same inner diameter as the third symmetrical transformer. However, the third transformer has different metal width for the primary winding $(2.2 \mu \mathrm{m})$ and the secondary winding $(5.2 \mu \mathrm{m})$. The spacing for the third one is still 3.8 $\mu \mathrm{m}$. The quality factor and inductance for the primary winding and the secondary winding are shown in Figures 9(a) and 9(b). The coupling coefficient and transmission gain are also shown in Figure 9(c). The coupling coefficients at $1 \mathrm{GHz}$ for the three 


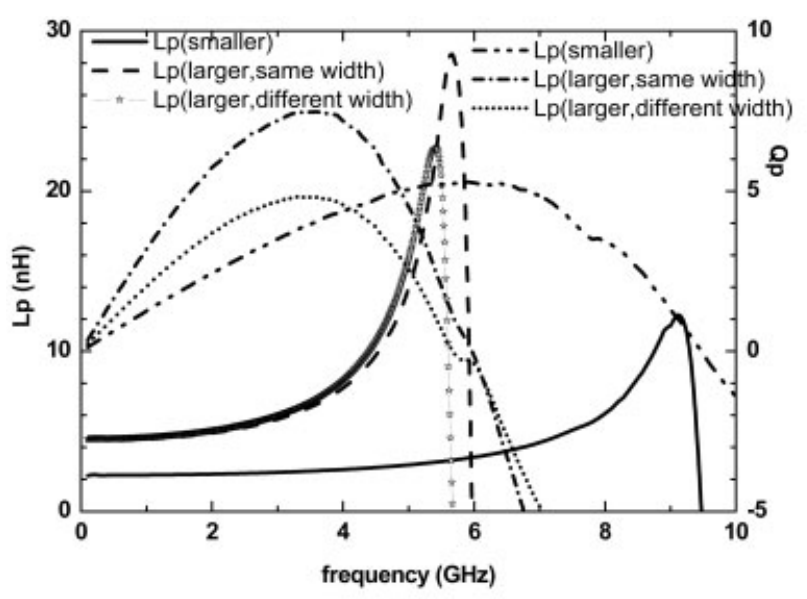

(a)

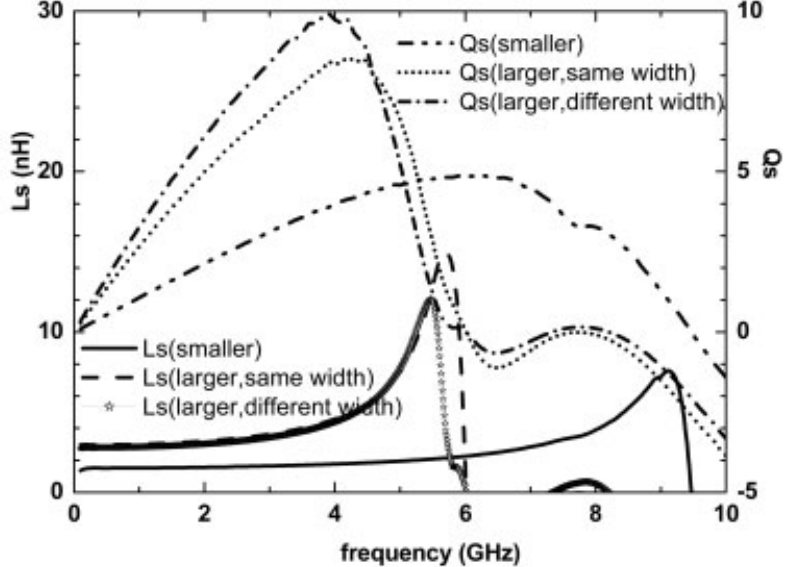

(b)

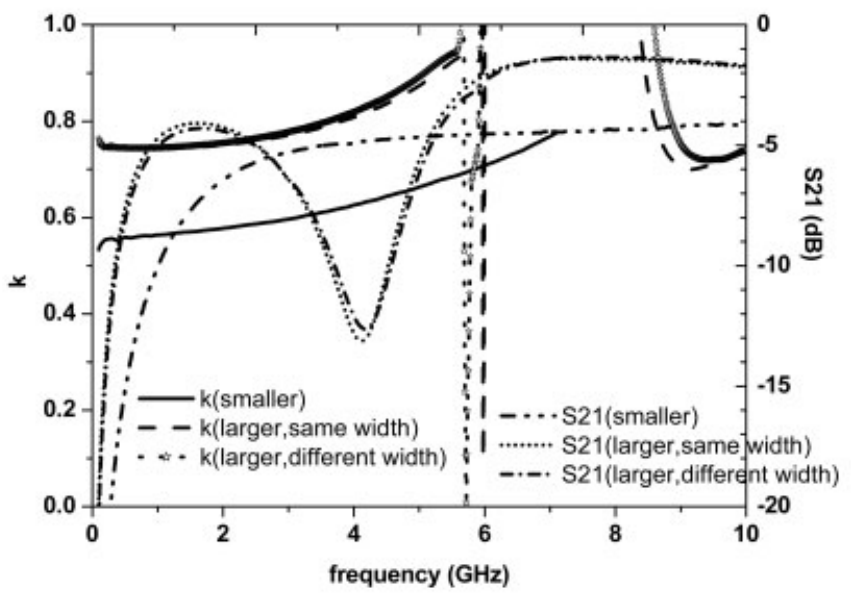

(c)

Figure 9 (a) Quality factor and inductance for the primary winding, (b) quality factor and inductance for the secondary winding, (c) coupling coefficient and transmission gain as a function of frequencies for three symmetrical transformers

TABLE 4 Symmetrical Transformer Results

\begin{tabular}{|c|c|c|c|c|c|}
\hline Type & $L_{\mathrm{p}}$ at $1 \mathrm{GHz}(\mathrm{nH})$ & $R_{\mathrm{p}}$ at $1 \mathrm{GHz}(\mathrm{Ohm})$ & $Q_{\text {pmax }}$ & $F$ at $Q_{\text {pmax }}(\mathrm{GHz})$ & $\mathrm{SRF}(\mathrm{GHz})$ \\
\hline I & 2.435 & 13.085 & 5.285 & 5.9 & 9.47 \\
\hline II & 4.515 & 8.897 & 7.471 & 3.6 & 5.97 \\
\hline \multirow[t]{2}{*}{ III } & 4.614 & 14.263 & 4.819 & 3.3 & 5.67 \\
\hline & $L_{\mathrm{s}}$ at $1 \mathrm{GHz}(\mathrm{nH})$ & $R_{\mathrm{s}}$ at $1 \mathrm{GHz}(\mathrm{Ohm})$ & $Q_{\text {smax }}$ & $F$ at $Q_{\mathrm{s}} \max (\mathrm{GHz})$ & $\mathrm{SRF}(\mathrm{GHz})$ \\
\hline I & 1.647 & 9.961 & 4.867 & 6.3 & 9.47 \\
\hline II & 3.012 & 7.094 & 8.465 & 4.46 & 6.02 \\
\hline III & 2.826 & 5.428 & 9.943 & 3.9 & 6.01 \\
\hline
\end{tabular}

symmetrical transformers are $0.66,0.74$, and 0.74 , respectively. Table 4 summarizes the results.

\section{CONCLUSIONS}

Four different types of the transformers, including stacked transformers, coupling stacked transformers, interleave transformers, and symmetrical transformers, are implemented on the GaAs substrate. In this article, the properties and trade-offs are discussed and the measurement data is also established to provide a useful design library for GaAs RFIC applications.

\section{ACKNOWLEDGMENTS}

This work is supported by the National Science Council of Taiwan, Republic of China, under contract numbers NSC 96-2752-E-009-
001-PAE, NSC 95-2221-E-009-043-MY3, by the Ministry of Economic Affairs of Taiwan under contract number 96-EC-17-A-05S1-020, and by MoE ATU Program under contract number 95W803. The authors thank the National Chip Implementation Center (CIC) for technical support.

\section{REFERENCES}

1. A.M. Niknejad and R.G. Meyer, Analysis, design, and optimization of spiral inductors and transformers for Si RF IC's, IEEE J Solid-State Circuits 33 (1998), 1470-1481.

2. C.P. Yue and S.S. Wong, On-chip spiral inductors with patterned ground shields for Si-Based RF IC's, IEEE J Solid-State Circuits 33 (1998), 743-752. 
3. J.R. Long, Monolithic transformers for silicon RF IC design, IEEE J Solid-State Circuits 35 (2000), 1368-1382.

4. T.O. Dickson, M.A. LaCroix, S. Boret, D. Gloria, R. Beerkens, and S. Voinigescu, 30-100-GHz inductors and transformers for millimeterwave (Bi)CMOS integrated circuits, IEEE Trans Microwave Theory Tech 55 (2007).

5. J.R. Long, A low-voltage 5.1-5.8-GHz image-reject downconverter RF IC, IEEE J Solid-State Circuits 33 (2005), 123-133.

6. M. Zannoth, B. Kolb, J. Fenk, and R. Weigel, A fully integrated VCO at $2 \mathrm{GHz}$, IEEE J Solid-State Circuits 33 (1998), 1987-1991.

7. N.M. Nguyen and R.G. Meyer, A $1.8-\mathrm{GHz}$ monolithic LC voltagecontrolled oscillator, IEEE J Solid-State Circuits 27 (1992), 444-450.

8. J. Crols and M. Steyaert, A single-chip $900 \mathrm{MHz}$ CMOS receiver front-end with a high performance low-IF topology, IEEE J SolidState Circuits 30 (1995), 1483-1492.

9. J.C. Rudell, J.-J. Ou, T.B. Cho, G. Chien, F. Brianti, J.A. Weldon, and P.R. Gray, A $1.9 \mathrm{GHz}$ wide-band IF double conversion CMOS integrated receiver for cordless telephone applications, IEEE J Solid-State Circuits 32 (1997), 2071-1088.

10. P. Andreani, A low-phase-noise, low-phase error, $1.8 \mathrm{GHz}$ quadrature CMOS VCO, in Proceedings of the ISSCC 2002, San Francisco, CA February 2002, pp. 290-291.

11. C.C. Meng, T.H. Wu, and M.C. Lin, Compact 5.2-GHz GaInP/GaAs HBT Gilbert upconverter using lumped rat-race hybrid and current combiner, IEEE Microwave Wireless Components Lett 15 (2005), $688-690$

12. T.-H. Wu, S.-C. Tseng, C. Meng, and G.-W. Huang, GaInP/GaAs HBT sub-harmonic Gilbert mixers using stacked-LO and leveled-LO topologies, IEEE Trans Microwave Theory Tech 55 (2007).

13. T.-H. Wu and C. Meng, 10-GHz highly symmetrical sub-harmonic Gilbert mixer using GaInP/GaAs HBT technology, IEEE Microwave Wireless Component Lett 17 (2007), 370-372.

14. T.-H. Wu and C. Meng, 5.2/5.7 GHz $48 \mathrm{~dB}$ image rejection GaInP/ GaAs HBT weaver down-converter using LO frequency quadrupler, IEEE J Solid-State Circuit 41 (2006), 2468-2480.

15. C.C. Meng, D.W. Sung, and G.W. Huang, A $5.2 \mathrm{GHz}$ GaInP/GaAs HBT double quadrature downconverter with polyphase filters for 40 dB image rejection, IEEE Microwave Wireless Components Lett 15 (2005), 59-61.

16. S.C. Tseng, C.C. Meng, C.K. Wu, and G.W. Huang, Low-voltage GaInP/GaAs HBT wideband Gilbert downconverter using transformer RF balun, 2007 IEEE MTT-S International Microwave Symposium, Honolulu, HI June 2007.

17. C.C. Meng, et al., $4.9 \mathrm{GHz}$ low-phase-noise transformer-based superharmonic-coupled GaInP/GaAs HBT QVCO, IEEE Microwave Wireless Components Lett (2006).

(c) 2008 Wiley Periodicals, Inc.

\section{ANALYSIS OF PROPAGATION LOSS OF METALLIC PHOTONIC CRYSTAL WAVEGUIDES}

\section{Yoshihiro Kokubo}

Graduate School of Engineering, University of Hyogo, 2167 Shosha, Himeji-shi, Hyogo 671-2201, Japan; Corresponding author:

kokubo@eng.u-hyogo.ac.jp

Received 21 March 2008

ABSTRACT: Metallic waveguides can be constructed with sidewalls consisting of metallic rods instead of sheets. The attenuation constants and bandwidths of such waveguides are calculated for a fixed cutoff frequency while the radii of the metallic rods are varied. The propagation losses are found to decrease when the radii of the rods is increased. (C) 2008 Wiley Periodicals, Inc. Microwave Opt Technol Lett 50: 2942-2945, 2008; Published online in Wiley InterScience (www. interscience.wiley.com). DOI 10.1002/mop.23844
Key words: microwave; waveguide; metallic photonic crystal; attenuation constant

\section{INTRODUCTION}

Electromagnetic waves with a frequency lower than some cutoff cannot propagate through a metal wire mesh. This principle is the basis for photonic crystals composed of a three-dimensional periodic structure of metallic wires [1] or the bandgap is calculated for a negative dielectric constant due to surface plasmon polaritons on the metal wire mesh [2]. The propagation modes in a waveguide whose walls are replaced with metal wires have been calculated [3]. A waveguide structure whose E-planes are replaced by metal wires can easily confine electromagnetic waves because the direction of the electric field is parallel to the wires. Recently, parallelplate postwall waveguides [4] and substrate-integrated waveguides (SIWs) [5] have been constructed, consisting of microstrip lines with only one row of metal wires on each side. A SIW is synthesized by forming two rows of metallic holes in a substrate. The field distribution in a SIW is similar to that in a conventional rectangular waveguide. In this article, the attenuation constants of metallic photonic crystal (MPC) waveguides, whose E-planes are replaced by arrayed metallic rods, are calculated and compared with those of conventional metallic waveguides.

\section{BASIC STRUCTURE}

The basic structure of the MPC waveguide is shown in Figure 1. It is composed of metal rods arranged in a triangular or square lattice. One or more rows are removed and they are sandwiched between parallel metal plates. Consequently, the E-planes have been replaced with MPCs while the H-planes have been left as is. Define a as the lattice constant of metal rods and $w$ as the gap width between the removed rods. For a triangular lattice, $w$ is narrower than that of a square lattice for the same value of $a$.

The field distribution in the waveguide is similar to that in a conventional rectangular waveguide and is uniform along the $y$-axis. Figure 2 shows a bandgap map for E polarization (in which the electric field is parallel to the axis of the metal rods) with varying normalized radius of the rods $r / a$. The areas enclosed by the oblique lines represent bandgaps where no propagation modes exist. We fixed the values of $r / a$ at $0.1,0.2$, and 0.3 for $w / a=2.5$ and removed one row of metal rods from them. The propagation modes for this waveguide were calculated using the supercell approach [6], by applying periodic Bloch boundary conditions to the unit cell [7]. The results are shown in Figure 3. The upper area of the bandgap is out of reach when $\omega a / 2 \pi c>0.42(r / a=0.1)$, $\omega a / 2 \pi c>0.6(r / a=0.2)$, and $\omega a / 2 \pi c>0.94(r / a=0.3)$ for triangular lattices; and when $\omega a / 2 \pi c>0.38(r / a=0.1), \omega a l$ $2 \pi c>0.52(r / a=0.2)$, and $\omega a / 2 \pi c>0.74(r / a=0.3)$ for square

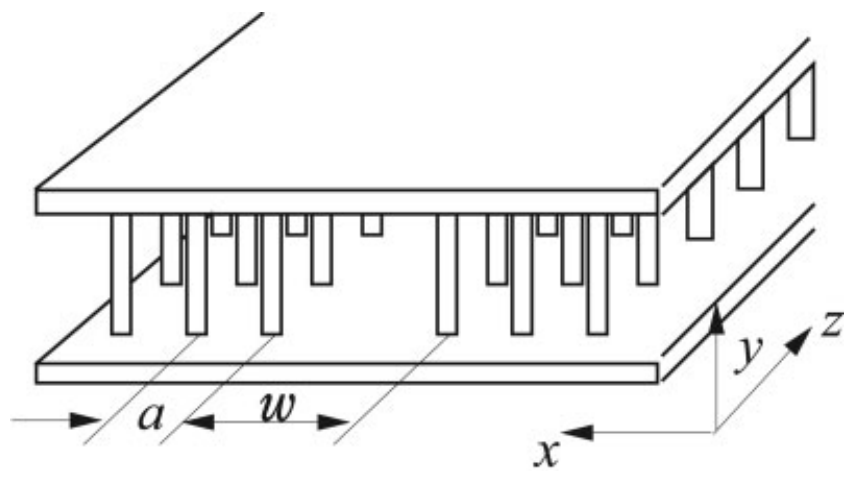

Figure 1 Perspective view of the MPC waveguide 